\title{
Modulation of Lingual Lipase Development by Glucocorticoid in the Rat
}

\author{
PING-CHEUNG LEE, MARK F. STRUVE, AND STEVEN L. WERLIN \\ Division of Gastroenterology, Department of Pediatrics, The Medical College of Wisconsin. \\ Milwaukee, Wisconsin 53226
}

\begin{abstract}
Lingual lipase in the rat is present in the neonatal period and undergoes developmental increase during postnatal life. To evaluate the role of glucocorticoid in the control of lingual lipase during development, suckling rats were adrenalectomized at $\mathrm{d} \mathbf{1 0}$ and various hormone replacements were performed. Adrenalectomy abolished the developmental increase of lingual lipase. Low doses of dexamethasone ( 0.2 and $0.5 \mu \mathrm{g} / 100 \mathrm{~g}$ body wt) restored the lingual lipase to near normal level in adrenalectomized animals. High doses of dexamethasone $(20 \mu \mathrm{g} / 100 \mathrm{~g}$ body wt), when given to similarly adrenalectomized animals, however, led to a reduction of lingual lipase levels. Inhibition by dexamethasone is through the action of the hormone inasmuch as the coadministration of RU38486, a glucocorticoid type II receptor antagonist, completely abolished the inhibitory action. Inhibition is also steroid specific, with dexamethasone and triamcinolone acetonide being more effective. The results suggest a unique bimodal regulation of lingual lipase by dexamethasone in the rat serous glands. Because of the possible importance of lingual lipase as an alternative enzyme for fat digestion in neonates, the inhibitory action of high doses of glucocorticoid on lingual lipase development may have important implications. The use of steroidal compounds in the hastening of lung maturation and treatment of inflammatory disease might conceivably compromise their lingual lipase development, hence their capacities of fat digestion and malabsorption in the same period. (Pediatr Res 29: 46-49, 1991)
\end{abstract}

Current concepts of lipid digestion involve a complex interaction of lipolytic enzymes and various cofactors found in the gastrointestinal tract. In adult animals, particularly in the rat and human, pancreatic lipase is the key enzyme for the digestion of lipids (1). Despite extremely low levels of pancreatic lipase in the neonatal period of both rats and humans (2), overall malabsorption of lipids is uncommon because of alternative sources of lipase. These include the bile salt-stimulated milk lipase (3) and the lingual lipase secreted by the serous glands of the tongue (4). It has been shown that the lipolytic activity of rat milk cannot account for the marked hydrolysis of fats in the rat stomach (5). The major enzyme that contributes to intragastric lipolysis is lingual lipase (6). In rats, lingual lipase undergoes significant changes in activity during postnatal life (7). The mechanism of control for the development of this lingual lipase is unknown.

Hormones, particularly thyroxine and glucocorticoid, have been shown to play pivotal roles in regulating the development

Received May 31, 1990; accepted August 27, 1990.

Correspondence: P. C. Lee, Department of Pediatrics. The Medical College of Wisconsin, MACC Fund Research Center, 8701 Watertown Plank Road, Milwaukee, WI 53226 of digestive enzymes in the gastrointestinal tract. Induction of enzymes has been demonstrated in the parotid gland (8), stomach (9), pancreas $(8,10)$, and small intestine $(11,12)$. The role of these hormones in the development of lingual lipase has not been investigated. The aim of our study was to examine if hormones, particularly glucocorticoid, were involved in the modulation of lingual lipase development in the neonatal rat.

\section{MATERIALS AND METHODS}

Animals. Pregnant Sprague-Dawley rats were housed in individual cages and maintained on a 12-h alternate light-dark cycle. On the expected date of delivery, cages were checked every $2 \mathrm{~h}$ for birth, and the day of birth was regarded as $d 0$. Pups were allowed to suckle freely until the time of sacrifice. All rats were killed by decapitation at the age stated. After sacrifice, the posterior half of the tongue was dissected followed by careful removal of the muscles. In some experiments, particularly the ones in which rats received dexamethasone treatment, pancreata were also collected from the same animals to serve as control tissues by evaluating their amylase and lipase activities as an index of in vivo response to glucocorticoid action. Isolated glandular tissues so obtained were either used immediately or stored frozen at $-70^{\circ} \mathrm{C}$. Preliminary studies showed that storage at $-70^{\circ} \mathrm{C}$ up to 1 mo did not result in appreciable loss of enzyme activity from either the tongue or pancreas. The NIH guidelines for the care and use of laboratory animals were followed for all protocols used to ensure that animals were not subjected to pain and discomfort.

Dexamethasone administration. Dexamethasone (Sigma Chemical Co., St. Louis, MO) was administered to rat pups at $12 \mathrm{~d}$ of age in the following concentrations: $0.5,1.0,10,25$, and $50 \mu \mathrm{g} / 100 \mathrm{~g}$ body weight (intraperitoneally), $48 \mathrm{~h}$ before sacrifice. Age-matched littermates were given a single injection of vehicle and served as controls.

Adrenalectomy and sham operation. This procedure was performed under Metofane (Pitman-Moore, Inc., Washington Crossing, NJ) anesthesia. Pups were operated on $10 \mathrm{~d}$ after birth. Bilateral adrenalectomy was performed through a dorsal incision under anesthesia using sterile conditions. Except for the time study, all animals were killed $5 \mathrm{~d}$ after operation (i.e. at $\mathrm{d} 15$ of age). After sacrifice, all adrenalectomized animals were examined for the presence of regenerating adrenal tissue. Only those showing no visible regeneration were included in the study. Steroid replacement was done by intraperitoneal injection and administered daily starting at $\mathrm{d} 11$ for $3 \mathrm{~d}$. Sera from adrenalectomized animals were also collected and corticosterone levels were measured to confirm the absence of active adrenal tissues.

Biochemical determinations. For the preparation of homogenate for enzyme and protein determinations, frozen tissue was partially thawed and minced in 10 volumes of ice cold water. Tongue tissue was homogenized with a Polytron for $30 \mathrm{~s}$ with the container immersed in crushed ice. Triton-X100 was added to the homogenate to a final concentration of $0.08 \%$. The 
homogenate was homogenized again with the Polytron. The resulting homogenate was allowed to sit in ice for $60 \mathrm{~min}$ with occasional stirring. The homogenate was then centrifuged at $1500 \times g$ for $6 \mathrm{~min}$. The supernatant was recovered and used for lingual lipase and protein determinations. Pancreatic tissues were homogenized with a Potter-Elvehjem homogenizer using a motor-driven pestle for $30 \mathrm{~s}$ with the vessel immersed in crushed ice. The homogenate was further sonicated for $5 \mathrm{~s}$ before being used for the determination of amylase, lipase, and protein. Lingual lipase was determined by potentiometric titration (at a constant $\mathrm{pH}$ of 5.0) of ionized fatty acids liberated from tributyrin $(20 \mathrm{mM})$ in a citrate-phosphate buffer $(1 \mathrm{mM})$ with $0.01 \%$ Triton-X100. Units were expressed as $\mu \mathrm{mol}$ of $\mathrm{NaOH}$ required to neutralize FFA liberated per min per $\mathrm{mg}$ of protein. Pancreatic amylase was determined by the saccharogenic method using soluble starch as the substrate (13). Units were expressed as $\mu \mathrm{mol}$ of maltose liberated per min per mg of protein. Pancreatic lipase activity was determined by potentiometric titration (at a constant $\mathrm{pH}$ of 8.0) of ionized fatty acids liberated from an olive oil emulsion with $0.2 \mathrm{~N} \mathrm{NaOH}$ (14). Units were expressed as $\mu \mathrm{mol}$ of $\mathrm{NaOH}$ required to neutralize FFA liberated per min per mg of protein. Protein was determined by the method of Lowry using BSA fraction V as the standard (15). Total corticosterone was measured by a competitive binding assay based on the method of Murphy (16), as modified by Bassett and Hinks (17). Charcoal adsorption was used to separate the bound and free ${ }^{3} \mathrm{H}$ corticosterone rather than gel filtration as reported previously (18).

Reagents. All reagents used for biochemical determinations were from Sigma Chemical Co. RU 38486 (U486) was a gift kindly provided by Dr. D. Martini of Roussel Uclaf, Romanville, France.

Statistics. Results are reported as the mean \pm SEM. Difference between the means of two groups were evaluated by $t$ test. For multigroup comparison, analysis of variance was used. A value of $p<0.05$ was considered significant.

\section{RESULTS}

Developmental profile of lingual lipase. At birth, the activity of lingual lipase was low. A gradual increase in activity was seen just before weaning at around $15 \mathrm{~d}$ of age (Fig. 1). A second increase in activity was evident after $15 \mathrm{~d}$ of age and eventually reached the activity of the adult level.

Effect of dexamethasone. Intact animals receiving a single dose of dexamethasone showed a dose-dependent decrease in lingual lipase activity (Fig. 2). At doses of $\leq 10 \mu \mathrm{g} / 100 \mathrm{~g}$ body weight, dexamethasone had no effect on lingual lipase activity. A signif-

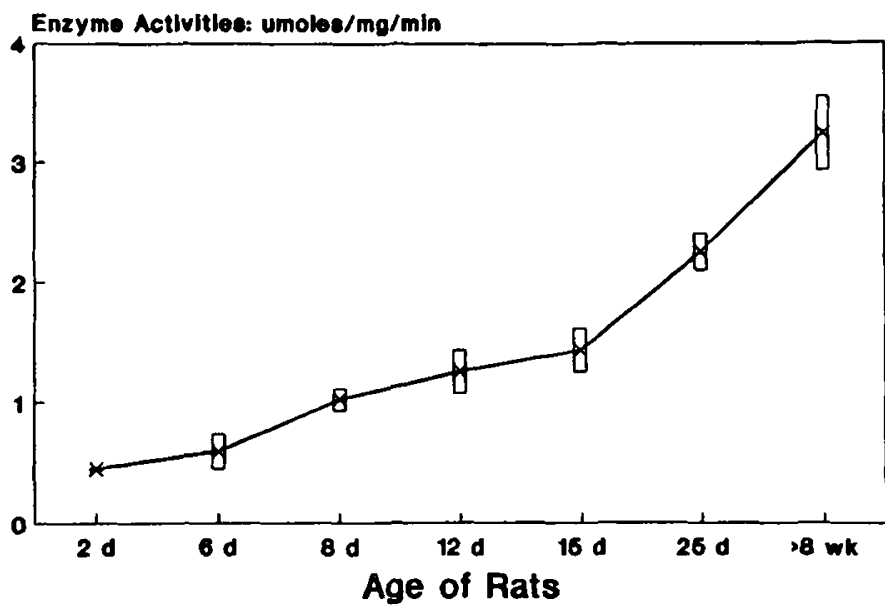

Fig. 1. Developmental profile of lingual lipase. The date of birth was considered as $\mathrm{d} 0$. Values represent the mean \pm SD from all the animals killed at each time point. The number varies from 4-30.

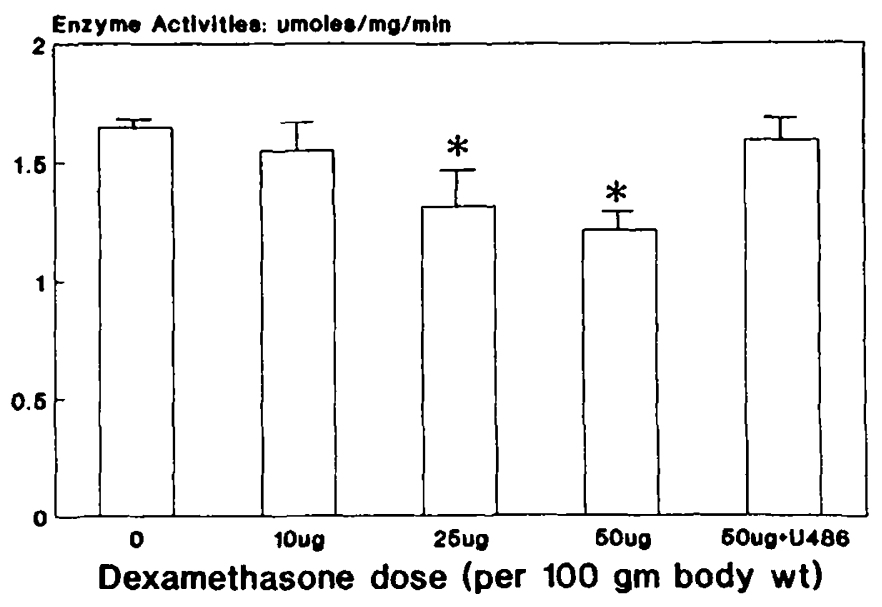

Fig. 2. Effect of varying concentrations of dexamethasone on lingual lipase activities in intact animals. Rat pups were given either vehicle $(0)$ or increasing dose of dexamethasone $(10,25$, and $50 \mu \mathrm{g} / 100 \mathrm{~g}$ body $w \mathrm{t})$ at $\mathrm{d} 12$ and were killed at $\mathrm{d} 14$. RU486 when used ( $3 \mathrm{mg} / 100 \mathrm{~g}$ body wt) was also given by intraperitoneal injection $1 \mathrm{~h}$ before and 12 and $24 \mathrm{~h}$ after dexamethasone administration. Values are mean of results of at least three animals from each group of treatment. Asterisks indicate values that were significantly different from the untreated group $(p<$ $0.05)$. The vertical lines above bars represent SD of the mean.

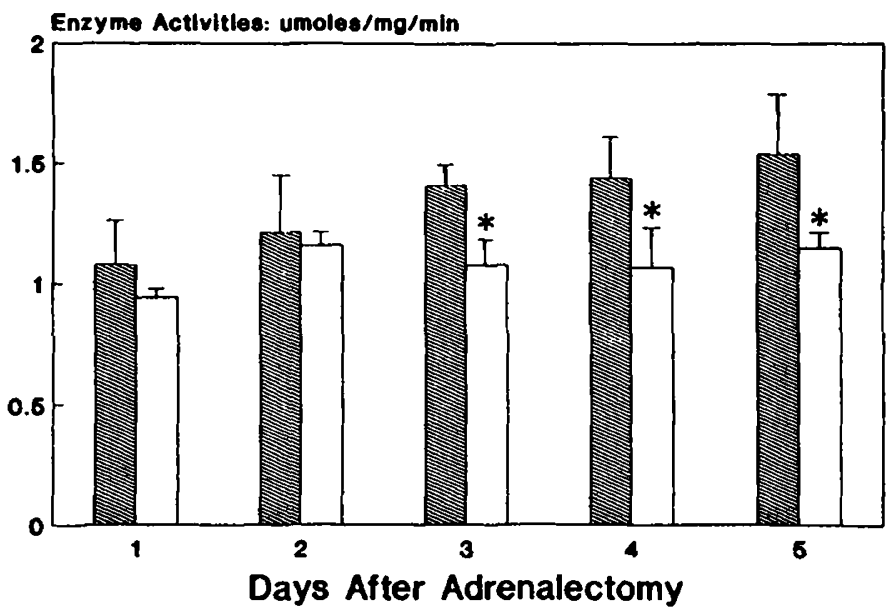

Fig. 3. Time course of lingual lipase activity after adrenalectomy. Rat pups were adrenalectomized at the age of $10 \mathrm{~d}$; lingual lipase was determined at $\mathrm{d} 1,2,3,4$, and 5 after surgery. Values are mean of results from at least three animals from each group at each time point. Asterisks indicate values that were significantly different from the control group of the same age $(p<0.05)$. The vertical lines above bars represent SD of the mean. Control, $\mathbb{Q}$; adrenalectomized $\square$.

icant decrease in activity was seen at $25 \mu \mathrm{g} / 100 \mathrm{~g}$ body weight. Further decrease in activity was shown at a higher dose of $50 \mu \mathrm{g} /$ $100 \mathrm{~g}$ body weight. To see if the action of dexamethasone was actually involved in the decrease in lingual lipase observed, U486, a known type II glucocorticoid receptor antagonist $(19,20)$, was also administered to a group of rats receiving $50 \mu \mathrm{g} / 100 \mathrm{~g}$ body weight of dexamethasone. Under the conditions studied, the decrease in lingual lipase activity was abolished by U486.

Effect of adrenalectomy. To see if glucocorticoid is required for the developmental increase in lingual lipase in the rat pups, pups were adrenalectomized at d 10 and their lingual lipase activity was followed on subsequent days. Figure 3 summarizes the results. In the control (sham operated) pups, lingual lipase activity showed the typical increase in activity with age. In contrast, the adrenalectomized littermates did not show the increase their counterparts did at the same age. The difference 
in lingual lipase activity became significant $3 \mathrm{~d}$ after adrenalectomy.

Effect of hormone replacement in adrenalectomized pups. To see if indeed the lack of glucocorticoid is the cause for the failure of lingual lipase to develop properly in the adrenalectomized animals, replacement studies were performed. Pups were adrenalectomized and dexamethasone was given at various doses. Figure 4 shows the results. At low concentrations of dexamethasone ( 0.2 and $0.5 \mu \mathrm{g} / 100 \mathrm{~g}$ body weight), an increase in lingual lipase activity over that of adrenalectomized littermates without supplementation was evident (Fig. 4A). At somewhat higher concentrations ( 1 and $5 \mu \mathrm{g}$ ), this effect disappeared. At even higher concentrations $(20 \mu \mathrm{g})$, a significant reduction in lingual lipase was seen. To confirm the action of dexamethasone in these experimental animals, their pancreata were also removed and checked for pancreatic amylase and lipase activity. Figure $4 B$ summarizes the results. As expected, both the lipase and amylase

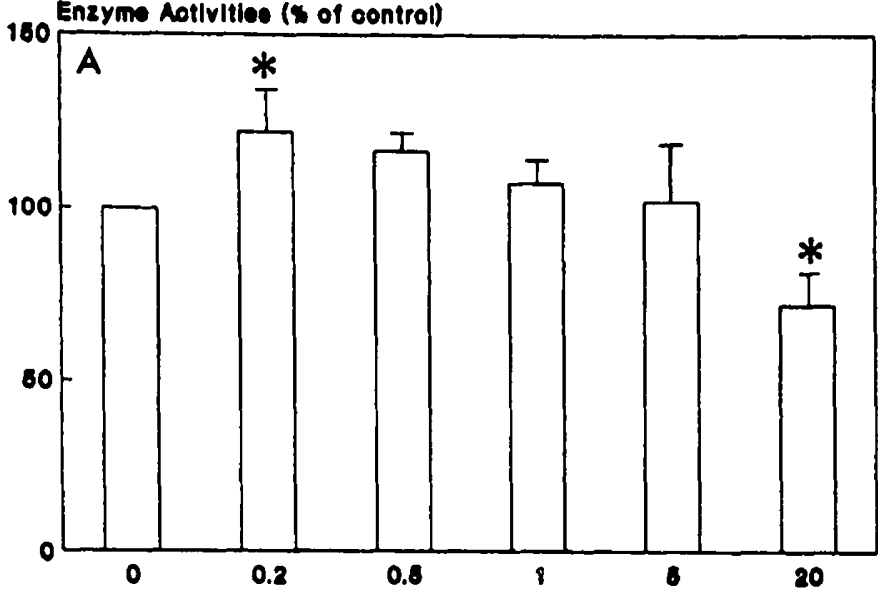

Enzyme Aotlvitlee (s of control)

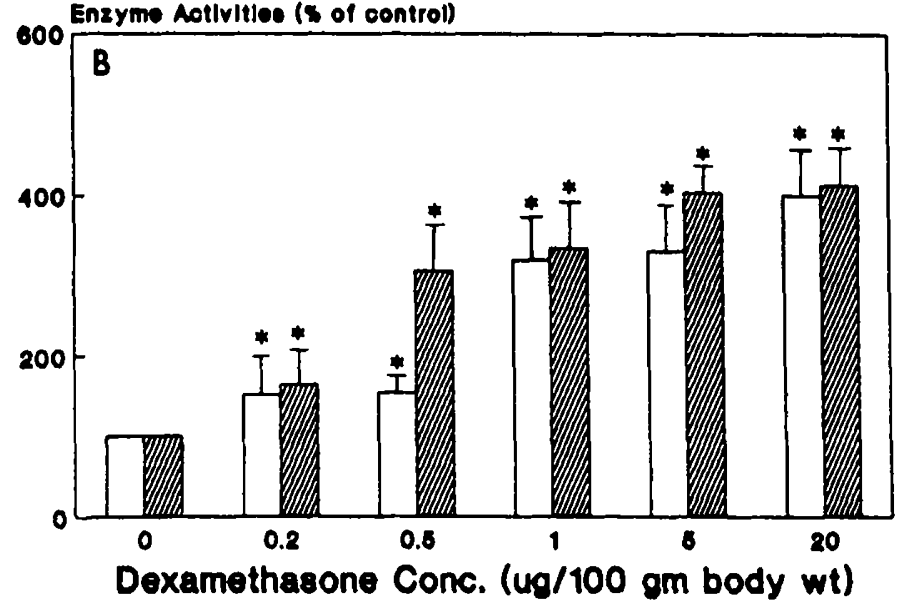

Fig. 4. Dosage effect of dexamethasone replacement on enzyme activity in adrenalectomized rat pups. Animals were adrenalectomized at the age of $10 \mathrm{~d}$. Dexamethasone at graded concentrations was given daily starting on d 12 until d 14. Animals were killed at d 15. Upper panel $(A)$ depicts the dosage effect on lingual lipase activity. Lower panel $(B)$ depicts that on pancreatic amylase (ש) and lipase $(\square)$ activity in the same animal. Results represent a composite of several experiments in which littermates were used for two or three different dose studies in each separate experiment. Values for each enzyme were then normalized against the activity (arbitrarily assigned as $100 \%$ ) of the same enzyme found in the controls, which were the adrenalectomized pups receiving only vehicle injection. Final values shown represent the mean of results from three to six animals for each group. Asterisks indicate values that were significantly different from the control group $(p<0.05)$. The vertical lines above the bars represent SD of the mean. activity showed a dose-dependent increase with increasing concentrations of administered dexamethasone.

To further examine the attenuation effect of steroids on lingual lipase activity during development, adrenalectomized pups were given high doses of various steroids and their effects were evaluated (Fig. 5). Of the five different steroids used, only two, i.e. dexamethasone and triamcinolone acetonide, were effective in attenuating the lingual lipase activities. Corticosterone acetate and aldosterone even at much higher doses had no effects on lingual lipase activity. Testosterone resulted in an increase of lingual lipase activity. The reduction in lingual lipase activity was not due to inhibition by the specific steroids inasmuch as addition of these steroids to the assay mixture did not affect the enzyme activity in vitro. Further, mixing of the homogenate from the dexamethasone-treated animal that showed a decrease in lingual lipase activity and homogenates from control animals with normal lingual lipase activity did not result in any observable inhibition.

\section{DISCUSSION}

Neonatal rats and humans both have insufficient amounts of pancreatic lipase for adequate digestion of dietary fats. Lingual lipase has been shown to be an important alternative source and serves as a compensatory mechanism in these neonates. Our study using a rat model shows a developmental profile of lingual lipase very similar to that reported by Hamosh and Hand (7). The enzyme is present at very low concentration in late-term fetal and in early postnatal life and increases at the time of weaning ( $15 \mathrm{~d}$ of age). These precise stepwise increases suggest a temporal event that is coordinated by specific cellular mechanisms. Hormones, particularly glucocorticoids and thyroxine, have been shown to be important regulators of digestive enzyme development. In salivary glands, a close temporal relationship exists between the changing patterns of parotid amylase, histology, and corticosterone levels in developing rat pups (21-23). Pharmacologic doses of steroids have been shown to stimulate parotid gland development in rodents (23-26). Suppression of corticosterone production by adrenalectomy depressed the

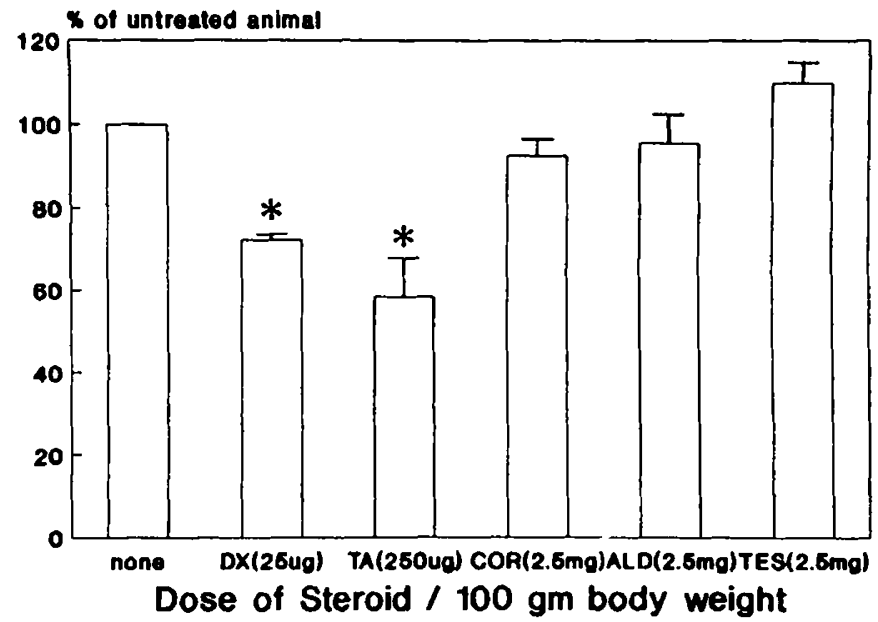

Fig. 5. Specificity of steroids in their effects on lingual lipase activity in adrenalectomized rat pups. Animals were adrenalectomized at the age of $10 \mathrm{~d}$. Various steroids at the concentrations indicated were given daily starting on d 12 until d 14. Animals were killed at d 15. Results represent a composite of several experiments in which littermates were used for two or three different steroids in each separate experiment. Values for the enzyme activity were then normalized against the activity of the controls (arbitrarily assigned as $100 \%$ ), which were the adrenalectomized pups receiving only vehicle injection. Final values shown represent means of results from three to six animals for each group. Asterisks indicate values that were significantly different from the control group $(p<0.05)$. The vertical lines above the bars represent SD of the mean. 
growth and amylase contents in the parotid glands (25). These results all point to the importance of glucocorticoid as a modulating factor for salivary gland development.

Our study shows that the glucocorticoid status of the developing pups also affects the level of lingual lipase. Unlike the inductive effect of glucocorticoid for almost all digestive enzymes, the regulation of lingual lipase by glucocorticoid is multifaceted and complex. Our results indicated that a hypocorticosteroid state as a result of adrenalectomy abolished the developmental increase of lingual lipase. Corticosteroid replacement by dexamethasone administration at a physiologically meaningful dose enables a normal development of lingual lipase in these pups, yet a pharmacologic dose of dexamethasone decreased lingual lipase compared with untreated adrenalectomized animals. Similar high doses of dexamethasone given to intact animals also decreased lingual lipase levels. The observed results are indeed due to the action of dexamethasone, inasmuch as coadministration of a glucocorticoid type II receptor antagonist (RU486) completely abolished the inhibitory effect of dexamethasone. Furthermore, the exocrine enzymes (amylase and lipase) in the pancreata from these same animals showed a typical response to the administered dexamethasone in that there were graded increases in both lipase and amylase levels with increasing amounts of dexamethasone received.

Our results further showed that the inhibitory action is specific in that some but not all steroids were effective. Only those with potent glucocorticoid action, in this case, dexamethasone and triamcinolone acetonide, were inhibitory. The naturally occurring glucocorticoid in the rat, corticosterone, however, has no effect at the concentration tested, 100 times that of dexamethasone, although an induction of pancreatic amylase was evident in similarly treated pups (data not shown). Testosterone, on the other hand, showed stimulation instead of inhibition at the concentration used.

As yet, we do not know the mechanism that leads to a decrease in lingual lipase after treatment with pharmacologic doses of dexamethasone. Dexamethasone did not cause the appearance of an inhibitor or activator for lingual lipase inasmuch as mixing experiments using tissue homogenates of control and dexamethasone-treated pups showed no reduction or stimulation in lingual lipase activity. It is likely that the control occurs at the level of enzyme synthesis. This remains to be investigated.

The dual action of dexamethasone as observed in our study is unique. Glucocorticoid has been shown in other tissues to have either an inductive or repressive effect but normally for different enzymes (27). The action of dexamethasone in the control of rat lingual lipase thus offers an interesting system and deserves to be examined further at the molecular level.

Our results show that the developmental increase of lingual lipase in the rat during postnatal life is dependent on adrenal function. One of the key hormones appears to be glucocorticoid inasmuch as dexamethasone at physiologic concentration can restore the level of lingual lipase in adrenalectomized pups. Recent reports present convincing evidence for the presence of a gastric lipase, which quantitatively may be more important in the overall digestion of fat in humans $(28,29)$. There is, however, indication that lingual lipase might be of greater importance in early life than in adulthood (30). If indeed this is the case, and if the lingual lipase system in human infants responded similarly, the inhibitory action of glucocorticoids at pharmacologic doses would have important implications because of a possible role of lingual lipase in fat digestion and absorption by neonates.

Acknowledgments. The authors thank Merle Farnham for his generous support. We also thank Becky Parise for her help in the preparation of the manuscript.

\section{REFERENCES}

1. Linder MC 1985 Nutrition and metabolism of rats. In: Linder MC (ed) Nutritional Biochemistry and Metabolism. Elsevier, New York, pp 33-50

2. Lebenthal E, Lee PC 1980 Development of functional responses in human exocrine pancreas. Pediatrics 66:556-560

3. Blackberg L, Hernell O 1983 Further characterization of the bile salt-stimulated lipase in human milk. FEBS Lett 157:337-341

4. Hamosh M 1979 The role of lingual lipase in neonatal fat digestion. In Harris JT (ed) Development of Mammalian Absorptive Process, Ciba Foundation Series 70. Elsevier, Amsterdam, pp 69-98

5. Hamosh M, Scow RO 1971 Lipoprotein lipase activity in guinea pig and rat milk. Biochim Biophys Acta 231:283-289

6. Hamosh M, Scow RO 1973 Lingual lipase and its role in the digestion of dietary lipid. J Clin Invest 52:88-95

7. Hamosh M, Hand AR 1978 Development of secretory activity in serous cells of the rat tongue. Dev Biol 65:100-113

8. Sasaki R, Mara M, Takeuchi T, Furihata C, Matsushima T, Sugimura T 1976 Premature induction of amylase in pancreas and parotid gland of growing rats by dexamethasone. Biochim Biophys Acta 428:619-626

9. Yahav J, Lee PC, Lebenthal E 1986 Ontogeny of pepsin secretory response to secretagogues in isolated rat gastric glands. Am J Physiol 250:6200-6204

10. Lee PC, Kratz B, Kim O, Moshier J, Lin CH 1990 Expression of the amylase gene in the rat exocrine pancreas during postnatal development: effect of dexamethasone. Biochim Biophys Acta 1049:244-248

11. Moog I, Denes AE, Powell PM 1973 Disaccharidase in the small intestine of the mouse: normal development and influence of cortisone, actinomycin $D$ and cycloheximide. Dev Biol 35:143-159

12. Jumawan J, Celano P, Horowitz C, Lau H, Koldovsky O 1977 Effect of cortisone or L-triiodothyronine administration to pregnant rats on the activity of fetal intestinal disaccharidases and lysosomal acid $\beta$-galactosidase. Biol Neonate 32:211-217

13. Searcy RL, Hayashi S, Belk JE 1956 A new microsaccharogenic method for serum amylase determination. Tech Bull Regist Med Technol 36:252-256

14. Smervia M, Dutour C, Desnnelle P 1971 On the probable involvement of a histidine residue in the active site of pancreatic lipase. Biochemistry 10:21432149

15. Lowry OH, Rosebrough NJ, Farr AL, Randall RJ 1951 Protein measurement with the Folin phenol reagent. J Biol Chem 193:265-275

16. Murphy BEP 1967 Some studies of the protein binding of steroids and their application to the routine micro and ultra-micro measurement of various steroids in body fluids by competitive protein binding radioassay. $\mathrm{J}$ Clin Endocrinol Metab 27:973-990

17. Bassett JM. Hinks NT 1969 Micro determination of corticosteroids in ovine peripheral plasma: effects of veni-puncture, corticotrophin, insulin and glucose. J Endocrinol 44:387-403

18. Lu RB. Lebenthal E, Lee PC 1987 Developmental changes of glucocorticoid receptors in the rat pancreas. J Steroid Biochem 26:213-218

19. Bertagna X, Bertagna C. Luton JP, Husson JM, Girard F 1984 The new steroid analog RU486 inhibits glucocorticoid action in man. J Clin Endocrinol Metab 59:25-28

20. Lazar G, Agaravol MK 1986 Physiological action and receptor binding of a newly synthesized and novel antiglucocorticoid. Biochem Biophys Res Commun 134:44-50

21. Redman RS, Sreebny LM 1976 Changes in patterns of feeding activity, parotid secretory enzymes and plasma corticostrone in developing rats. J Nutr 106:1295-1306

22. Takeuchi T, Ogawa M, Furihata C, Kawachi T, Sugimura T 1977 Perinatal changes in amylase and serum corticosterone levels in rats. Biochim Biophys Acta 497:657-602

23. Takuma T, Nakanishi M, Takagi Y, Yanemura T, Kumegawa M 1978 Precocious differentiation of mouse parotid glands and pancreas induced by hormones. Biochim Biophys Acta 538:376-383

24. Kumegawa M, Takuma T, Hosoda S, Nakanishi M 1987 Effects of hormones in differentiation of parotid glands of suckling mice in vitro. Biochim Biophys Acta 544:53-61

25. Kumegawa M, Maeda N, Yajima T, Takuma T, Ikeda I, Minamide C 1980 L-Thyroxine, cortisol and diet affect the level of amylase in the parotid gland of developing rats. J Endocrinol 87:65-71

26. Johnson DA, Etzel KR, Alvares OF, Cortez JE 1987 Regulation of parotid salivary proteins by glucocorticoids. J Dent Res 66:1563-1568

27. Burnstein KL, Cidlowski JA 1989 Regulation of gene expression by glucocorticoids. Ann Rev Physiol 51:683-699

28. DeNigris SJ, Hamosh M, Kasbekar DK, Lee TC, Hamosh P 1988 Lingual and gastric lipases: species differences in the origin of prepancreatic digestive lipases and the localization of gastric lipase. Biochim Biophys Acta 959:3845

29. Moreau H, Gargouri Y, Lecat D, Junien JL, Verger R 1988 Purification, characterization and kinetic properties of the rabbit gastric lipase. Biochim Biophys Acta 960:286-293

30. Salzman-Mann C, Hamosh M, Sivasubramanian KN, Bar-Maor A, Zinder O, A very GB, Watkins JB, Hamosh P 1982 Congenital esophageal atresia: lipase activity is present in the esophageal pouch and stomach. Dig Dis Sci 27:124128 